\title{
Modeling and Parameter Extraction of PV Cell Using Single- and Two-Diode Model
}

\author{
B. Benabdelkrim ${ }^{1,2}$, A. Benatillah ${ }^{2}$ \\ ${ }^{1}$ Department of Material Sciences, Institute of Science and Technology, University of Ahmed Draia, Adrar, ALGERIA \\ ${ }^{2}$ Laboratory of Energy, Environment and Systems of Information (LEESI), University Ahmed Draia Adrar, ALGERIA \\ benaekbouchra01@gmail.com
}

\begin{abstract}
Photovoltaic modules operate under a large range of conditions. This combined with the fact that manufacturers provide electrical parameters at specific conditions (STC). The present study proposes a comparison between single and double diode models of solar PV system and ensures the best suited model under specific environmental condition for accurate performance prediction. An important feature of these models is that its parameters can be determined using data commonly provided by module manufacturers on their published datasheets. Accurate determination of these parameters which arose from a diversification of models and methods dedicated to their estimations is still a challenge for researchers. In this paper the single and two diode models have been studied by mathematical methods based on simulated Newton-Raphson iteration method. Newton-Raphson iteration method is solved by MATLAB simulation.
\end{abstract}

Keywords: PV Module; Single-diode model; Two diode model; Performance I-V Curves, Parameter Extraction

Received: 10/12/2017 - Accepted: 25/12/2017

\section{Introduction}

The rapid growth of PV system utilizations is due to its availability everywhere which avoids transmission costs and losses, free, abundant and pollution free. Silicon is the basic material required for the production of solar cells based crystalline or thin film technology.

The photovoltaic (PV) modules are generally rated under standard test conditions (STC) with the solar radiation of $1000 \mathrm{~W} / \mathrm{m} 2$, cell temperature of $25^{\circ} \mathrm{C}$, and solar spectrum of 1.5 by the manufacturers. The parameters required for the input of the PV modules are relying on the meteorological conditions of the area. The climatic conditions are unpredictable due to the random nature of their occurrence. These uncertainties lead to either over- or underestimation of energy yield from PV modules. An overestimation up to $40 \%$ was reported as compared to the rated power output of PV modules [1,2]. The growing demand of photovoltaic technologies led to research in the various aspects of its components from cell technology to the modeling, size optimization, and system performance [3-5].

There are various PV cell modules studied by researchers in the literature. One of the simplest is single diode model. [6] In broad sense this model is derived by three parameters: Short Circuit Current $\left(\mathrm{I}_{\mathrm{sc}}\right)$, Open Circuit Voltage $\left(\mathrm{V}_{\mathrm{oc}}\right)$, and Diode Ideality Factor $(A)$. When the parameter series resistance $\left(R_{\mathrm{s}}\right)$ is added in this model, the accuracy of model gets improved. One drawback of this model is that it is not capable of temperature (T) variation handling. Parameter shunt resistance $\left(\mathrm{R}_{\mathrm{sh}}\right)$ significantly improves the model efficiency. [7] This model is having a drawback of reduced accuracy under low irradiance $(\mathrm{G})$ level, especially at open circuit voltage $\left(\mathrm{V}_{\mathrm{oc}}\right)$. Additional diode design is added to the model for the recombination loss in the depletion region of the cell of solar module. [8] This is double- diode model. This model has more parameters to calculate. This model gives more accuracy because this model is more practical especially under low voltages.

In this paper, a comparative analysis details the behavioral I-V characteristics of a single-diode using analytical four and five parameter model and twodiode model. The accuracy of the simulation results is verified by comparing it with published data provided by manufacturers of six PV modules of different types (mono-crystalline, poly-crystalline and thin-film).

\section{Mathematical models of PV module}

\section{II.1 Single-diode model}

An electrical circuit with a single diode (single exponential) is considered as the equivalent 
photovoltaic cell in the present article. Two different models drawn from the equivalent electrical-circuit are studied: namely four- and five-parameter models.

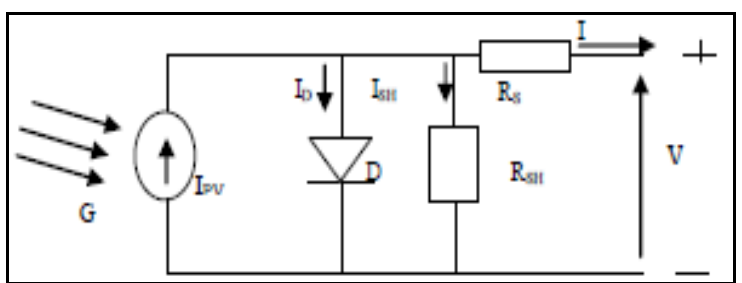

Figure1. PV-cell equivalent-circuit models: single-diode model [9].

An output current equation of I-V characteristic using this model can be written as:

$I=I_{p v}-I_{0} \cdot\left(\exp \left(\frac{V+R_{S} . I}{V_{T}}\right)-1\right)-\left(\frac{V+R_{S} . I}{R_{s h}}\right)$

Where

$I_{p v} \quad$ Photocurrent

$I_{0} \quad$ Cell saturation current

$R_{s h} \quad$ Shunt resistance

$R_{S} \quad$ Series resistance

$V_{T} \quad$ the thermal voltage $\left(\mathrm{V}_{\mathrm{T}}=\right.$ a.Ns.k.T/q $)$

$N_{s} \quad$ Number of cells in series

a Ideal factor of the PV diode

$q \quad$ Electron charge $\left(1.60281 \times 10^{-19} \mathrm{C}\right)$

$k \quad$ Boltzmann's constant $=1.38066 \times 10^{-23} \mathrm{~J} / \mathrm{K}$

$T \quad$ Cell operating temperature

\section{II.1.1 Four-parameter model}

The four-parameter model studied in this work has been used elsewhere [10, 11]. Assuming $\mathrm{R}_{\mathrm{sh}}$ as infinite and neglecting it in Equation (1), the fourparameter model is obtained as follows:

$I=I_{p v}-I_{\mathrm{O}} \cdot\left(\exp \left(\frac{V+R_{S} \cdot I}{V_{T}}\right)-1\right)$

The unknown parameters are denoted at STC as $I_{p v n}, I_{0 n}, \mathrm{a}_{n}$ and $R_{s n}$; where the " $\mathrm{n}$ " subscript refers to the reference operating conditions. The short circuit current can be found when $\mathrm{V}=0$

$$
I_{\text {scn }}=I_{\text {pvn }}
$$

The following equations are used to calculate the other parameters at STC [10].

$$
\begin{aligned}
& a_{n}= \frac{K_{v}-\frac{V_{o c n}}{T_{n}}}{V_{T n} \cdot\left(\frac{K_{i}}{I_{p v n}}-\frac{3}{T_{n}}-\frac{E_{g}}{k T_{n}^{2}}\right)} \\
& I_{\mathrm{on}}=\frac{I_{p \nu n}}{\exp \left(\frac{V_{o c n}}{V_{T n}}\right)-1}
\end{aligned}
$$

$$
R_{S n}=\frac{V_{T n} \cdot \ln \left(1-\frac{I_{m p n}}{I_{p v n}}\right)+\mathrm{V}_{o c n}-V_{m p n}}{\mathbf{I}_{m p n}}
$$

Where $\mathrm{Eg}$ is the band gap of the material The parameters can be found at any other operating conditions by using following equations:

$$
\begin{aligned}
& I_{p v}=\frac{G}{G_{n}}\left[I_{p v n}+K_{i}\left(T-T_{n}\right)\right] \\
& I_{\mathrm{O}}=I_{\mathrm{O} n}\left(\frac{T}{T_{n}}\right)^{3} \exp \left[\left(\frac{q \cdot E_{g}}{a \cdot k}\right)\left(\frac{1}{T_{n}}-\frac{1}{T}\right)\right] \\
& R_{s}=R_{S n} \\
& a=a_{n} \cdot \frac{T}{T_{n}}
\end{aligned}
$$

This model is implemented as follows: Eqs. (3)-(6) are used to find values of the four parameters under reference conditions. These four parameters are corrected for environmental conditions using Eqs. (7)-(10) and used in Eq. (2), which relates cell current to cell voltage. From Eq. (2) either cell current or voltage could be calculated provided that the other is known. Alternatively, cell current and voltage could both be calculated at the maximum-power point.

\section{II.1.2 Five-parameter model}

As given in Eq. (1), the five-parameter model is an implicit non-linear equation, which can be solved with a numerical iterative method such as Newton Raphson method [12]. However, this requires a close approximation of initial parameter values to attain convergence. Alternatively, the parameters may be extracted by means of analytical methods. Some of the analytical methods are studied elsewhere [12-15]. The five parameters $I_{p v}, I_{o}, R_{s}, R_{s h}$, and $m$ are calculated at a particular temperature and solarirradiance level from the limiting conditions of $\mathrm{V}_{\mathrm{oc}}$, $I_{\mathrm{sc}}, V_{\mathrm{mp}}, \mathrm{I}_{\mathrm{mp}}$ and using the following definitions of $\mathrm{R}_{\mathrm{so}}$ and $\mathrm{R}_{\text {sho }}$ :

$$
\begin{aligned}
& R_{s \mathrm{O}}=-\left.\frac{d V}{d \boldsymbol{I}}\right|_{V=V_{o c}} \\
& \boldsymbol{R}_{s h \mathrm{O}}=-\left.\frac{d V}{d \boldsymbol{I}}\right|_{I=I_{s c}}
\end{aligned}
$$

Where $R_{s 0}$ and $R_{s h 0}$ are the reciprocals of the slopes at the open-circuit point and short-circuit point, respectively. The values of these resistances are not usually provided by module manufacturers. The other parameters are calculated as follows. The following equations are used to calculate the five parameters required.

$$
I_{p v}=I_{s c}\left(1+\frac{R_{s}}{R_{s h}}\right)+\mathrm{I}_{0} \cdot\left(\exp \left(\frac{I_{s c} \cdot R_{s}}{V_{T}}\right)-1\right)
$$


$I_{0}=\left(I_{s c}-\frac{V_{o c}}{R_{s h}}\right) \cdot \exp \left(-\frac{V_{o c}}{V_{T}}\right)$

The value of the diode ideality factor (a) may be arbitrarily chosen. Many authors discuss ways to estimate the correct value of this constant.

Usually, $1 \leq \mathrm{a} \leq 2$ and the chosen value depend on other parameters of the I-V model. As it's given in [16], there are different opinions about the best way to choose (a). Because (a) expresses the degree of ideality of the diode and it is totally empirical, any initial value of ( a) can be chosen in order to adjust the model.

The $R_{s}$ and $R_{s h}$ resistances are calculated by iterative methods. The relation between $R_{s}$ and $R_{s h}$, may be found by making the maximum power calculated by the $\mathrm{I}-\mathrm{V}$ model, equal to the maximum experimental power from the datasheet $\left(\mathrm{P}_{\max , \mathrm{m}}=\right.$ $\left.\mathrm{P}_{\text {max,e }}\right)$ at the $\left(\mathrm{V}_{\mathrm{m}} ; \mathrm{I}_{\mathrm{m}}\right)$ point. In the iterative process, $R_{s}$ must be slowly incremented starting from $R_{s}=0$ and for every iteration, the value of $R_{s h}$ is calculated simultaneously:

$P_{\max , m}=V_{m p} \times\left\{I_{p v}-I_{0} \cdot\left(\exp \left(\frac{V_{m p}+R_{s} \cdot I_{m p}}{V_{T}}\right)-1\right)-\left(\frac{V_{m p}+R_{s} \cdot I_{m p}}{R_{s h}}\right)\right\}=P_{\text {max }, \mathrm{e}}$

$R_{s}=R_{s 0}-\frac{V_{T}}{I_{0}} \exp \left(-\frac{V_{o c}}{V_{T}}\right)$

$R_{s h}=\frac{V_{m p}+R_{s} \cdot I_{m p}}{I_{p v}-I_{0} \cdot\left(\exp \left(\frac{V_{m p}+R_{s} \cdot I_{m p}}{V_{T}}\right)-1\right)-\frac{P_{\max , e}}{V_{m p}}}$

The initial condition for the shunt resistance $R_{\text {sh }}$ can be found when considering the initial value of $\mathrm{R}_{\mathrm{s}}=0[17,18]$

$\boldsymbol{R}_{\mathrm{sh}, \min }=\frac{\boldsymbol{V}_{m p}}{\boldsymbol{I}_{s c}-\boldsymbol{I}_{m p}}-\frac{\boldsymbol{V}_{o c}-\boldsymbol{V}_{m p}}{\boldsymbol{I}_{m p}}$

In the proposed iterative method, the series resistance must be slowly incremented starting from a null value. Adjusting the I-V curve to match the cell reference condition requires finding the curve for several values of series and equivalent shunt resistances. The Newton-Raphson method was used in the proposed iterative method due to the ability to overcome undesired behaviors [19].

\section{II.2 Two-diode model}

The two diode model (Fig.2) equation of the I-V curve is expressed as [20]:

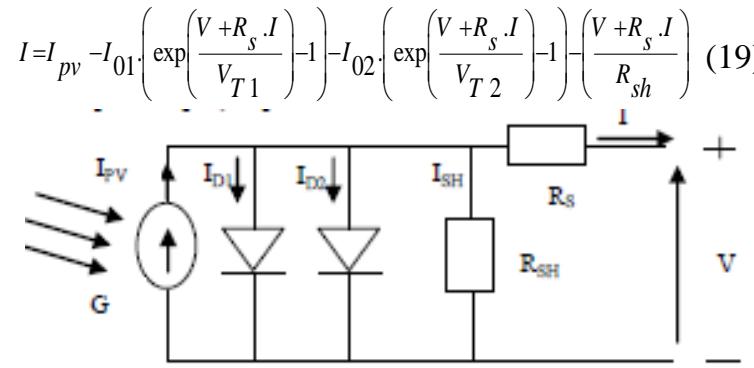

Figure2. PV-cell equivalent-circuit models: two-diode model

Where the diode factors $\mathrm{a}_{1}=1$ and $\mathrm{a}_{2}$ can be derived from:

$\frac{a_{1}+a_{2}}{p} \geq 1$

Where, $\mathrm{p}$ can be chosen greater than 2.2.

The rest of parameters can be deduced from the following equations [20]:

$$
\begin{aligned}
& I_{p v}=I_{s c} \\
& I_{01}=I_{02}=\frac{I_{s c}+K_{i} \cdot \Delta T}{\exp \left(\frac{q \cdot\left(V_{o c}+K_{v} \cdot \Delta T\right)}{k T \cdot\left(a_{1}+a_{2}\right) / \mathrm{p}}\right)-1}
\end{aligned}
$$

Rs and $\mathrm{R}_{\mathrm{sh}}$ are calculated by iterative method, similar to the procedure proposed by [21], where the relation between $R_{s}$ and $R_{s h}$ is chosen to verify that the calculated maximum power is equal to the experimental one $\left(\mathrm{P}_{\max , \mathrm{m}}=\mathrm{P}_{\max , \mathrm{e}}\right)$ at $\left(\mathrm{V}_{\mathrm{m}}, \mathrm{I}_{\mathrm{m}}\right)$ point. The $R_{s}$ value is found by a slow incrementation by the same manner as the above subsection.

The expression of $\mathrm{R}_{\mathrm{sh}}$ can be written as:

$$
R_{s h}=\frac{V_{m p}+R_{s} \cdot I_{m p}}{\left.I_{p v}-I_{01} \cdot \exp \left(q \frac{V_{m p}+R_{s} \cdot I_{m p}}{k T}\right)+\exp \left(q \frac{V_{m p}+R_{s} \cdot I_{m p}}{(\mathrm{p}-1) k T}\right)+2\right)-\frac{P_{\text {max,e }}}{V_{m p}}}
$$

\section{Results and discussion}

The modeling methods described in this paper are validated by measured parameters of selected PV modules. The experimental $(\mathrm{V}, \mathrm{I})$ data are extracted from the manufacturer's datasheet. Three different modules of different brands/ models are utilized for verification; these include the multi- and mono crystalline as well as thin-film types. The specifications of these modules are summarized in Table 1 
Table1. Specification of the PV modules

\begin{tabular}{|c|c|c|c|c|c|c|c|}
\hline Modules & $\begin{array}{l}\text { Isc } \\
\text { (A) }\end{array}$ & $\begin{array}{l}\text { Voc } \\
\text { (V) }\end{array}$ & $\begin{array}{l}\text { Imp } \\
\text { (A) }\end{array}$ & $\begin{array}{l}\text { Vmp } \\
\text { (V) }\end{array}$ & $\begin{array}{l}\mathbf{K i}(\mathbf{I s c}) \\
\left(\mathbf{m A} /{ }^{\circ} \mathbf{C}\right)\end{array}$ & $\begin{array}{l}\mathrm{Kv}(\operatorname{Voc}) \\
\left(\mathbf{m V} /{ }^{\circ} \mathbf{C}\right)\end{array}$ & Ns \\
\hline \multicolumn{8}{|l|}{ Poly-cristallin } \\
\hline$\overline{\text { Kyocera KC200GT }}$ & 8.21 & 32.9 & 7.61 & 26.3 & 3.18 & -123 & 54 \\
\hline Shell S70 & 4.5 & 21.2 & 4.12 & 17 & 2 & -76 & 36 \\
\hline$\frac{\text { Mono-cristallin }}{\text { Shell SQ150 }}$ & 4.8 & 43.4 & 4.4 & 34 & 1.4 & -161 & 72 \\
\hline Shell SP70 & 4.7 & 21.4 & 4.25 & 16.5 & 2 & -76 & 36 \\
\hline Thin-Film & & & & & & & \\
\hline Shell ST40 & 2.68 & 23.3 & 2.41 & 16.6 & 0.35 & -100 & 36 \\
\hline PVL-136 & 5.1 & 46.2 & 4.1 & 33 & 5.1 & -176 & 66 \\
\hline
\end{tabular}

Figures (3-5) shows the I-V curves for modules for different levels of irradiance and temperature. It can be seen that for varying irradiance, despite the modeling curves do not match experimental data in all points, the tow diode model strongly agrees to experimental data than the four-parameter and fiveparameter models for all types of modules, except for the thin-film (ST40) module at low irradiance of about $200 \mathrm{~W} / \mathrm{m}^{2}$ where the five-parameter modeled

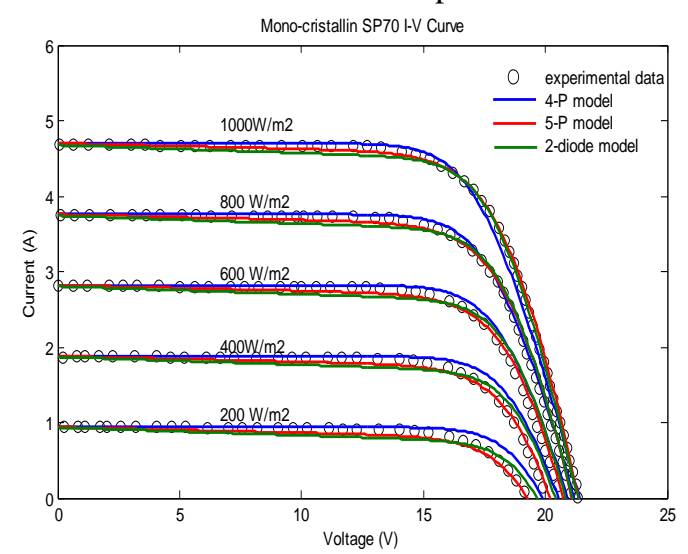

curve is closer to the experimental data than fourparameter and tow diode models.

In the case of the varying temperature, $G$ is kept constant at $1000 \mathrm{~W} / \mathrm{m}^{2}$. It can be noted that all three methods show good general agreement with the experimental data. However, a close inspection reveals that the tow-diode model yields the most accurate results at all temperature.

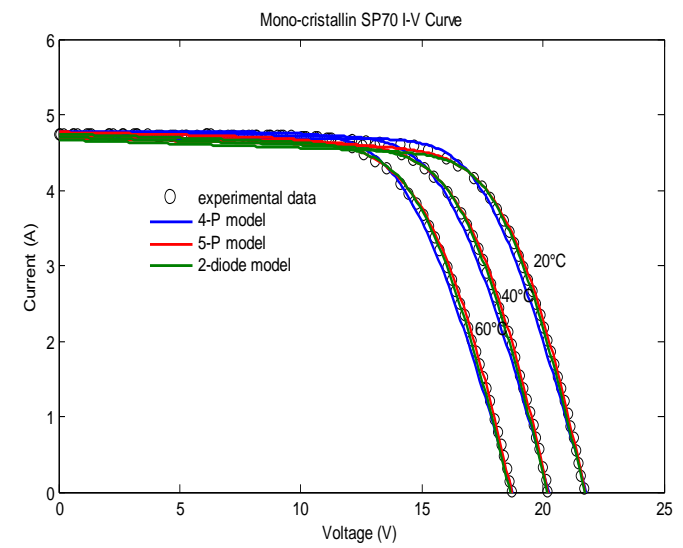

Figure3. The I-V characteristics of SP70 module at varying irradiance and temperature.
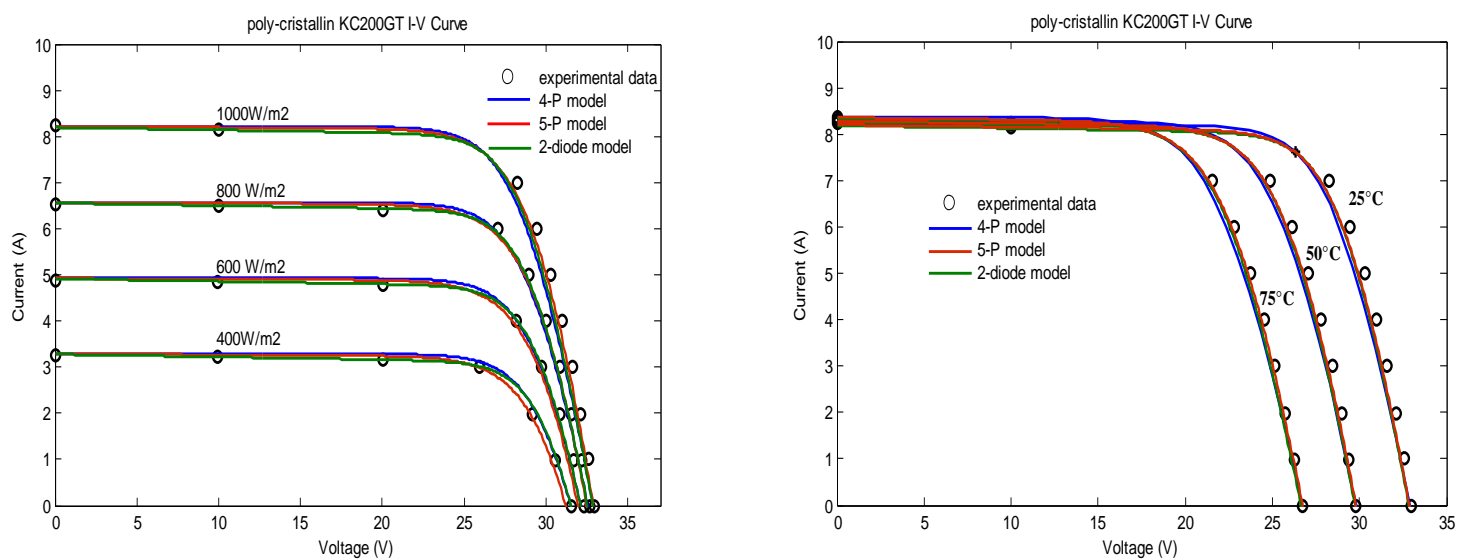

Figure4. The I-V characteristics of KC200GT module at varying irradiance and temperature. 

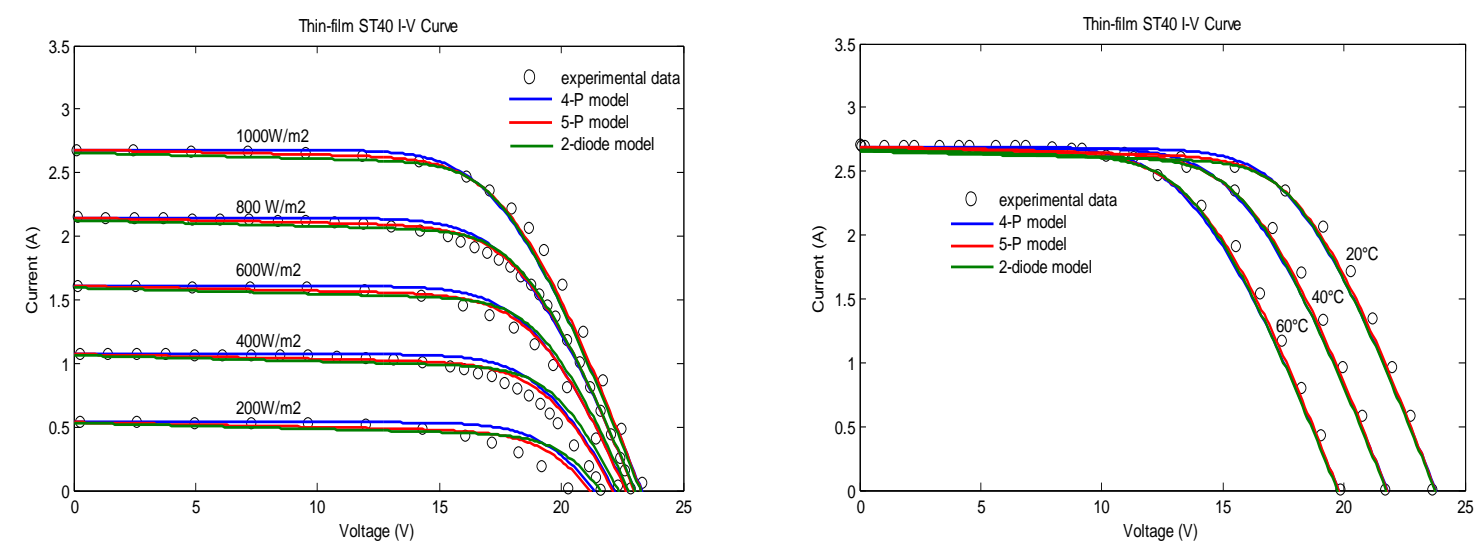

Figure5. The I-V characteristics of ST40 module at varying irradiance and temperature.

Table 2-4 shows the parameters used for three models. Four parameters are calculated namely, $\mathrm{I}_{0}$, $\mathrm{I}_{\mathrm{PV}}$, ideality factor (a) and Rs for the 4-P model. In the five parameter model, the additional calculated parameter is the shunt resistance; Rsh. and the twodiode model has more variables, the actual number of parameters computed is four because $\mathrm{I}_{01}=\mathrm{I}_{02}=\mathrm{I}_{0}$ while $a 1=1$ and $p$ can be chosen arbitrarily, i.e. $p \geq 2.2$. The two-diode model and the five parameter model exhibit similar results at STC. This is to be expected because both models use the similar max power matching algorithm to evaluate the model parameters at STC. However, at low irradiance, more accurate results are obtained from the two-diode model.

Table2.Parameters extracted for the four parameter model

\begin{tabular}{|c|c|c|c|c|c|c|}
\hline & \multicolumn{2}{|c|}{ Poly-crystalline } & \multicolumn{2}{|c|}{ Mono-crystalline } & \multicolumn{2}{|c|}{ Thin-Film } \\
\hline Module & KC200GT & S70 & SP70 & SQ150-PC & ST40 & PVL-136 \\
\hline$I p v$ & 8.2100 & 4.5000 & 4.7000 & 4.8000 & 2.6800 & 5.1000 \\
\hline$a$ & 1.0758 & 1.0177 & 1.0222 & 1.0594 & 1.3219 & 1.2573 \\
\hline$R s$ & 0.3541 & 0.4547 & 0.6310 & 1.0296 & 1.6156 & 2.3723 \\
\hline Io & $2.1954 \mathrm{e}-9$ & $7.4460 \mathrm{e}-10$ & $6.9528 \mathrm{e}-10$ & $1.1570 \mathrm{e}-9$ & $1.4202 \mathrm{e}-8$ & $1.9783 \mathrm{e}-9$ \\
\hline
\end{tabular}

Table3. Parameters extracted for the five parameter model

\begin{tabular}{l|llllll}
\hline & \multicolumn{3}{|c}{ Poly-crystalline } & \multicolumn{2}{c}{ Mono-crystalline } & \multicolumn{2}{c}{ Thin-Film } \\
\hline Module & KC200GT & S70 & SP70 & SQ150-PC & ST40 & PVL-136 \\
$I p v$ & 8.2146 & 4.5055 & 4.7150 & 4.8073 & 2.6961 & 5.2942 \\
$a$ & 1.3000 & 1.3000 & 1.3000 & 1.3000 & 1.3000 & 1.3000 \\
$R s$ & 0.2300 & 0.2200 & 0.4000 & 0.6700 & 1.5100 & 1.6800 \\
$R s h$ & 601.3368 & 189.0262 & 133.1309 & 466.4639 & 266.5478 & 44.1667 \\
Io & $9.8252 \mathrm{e}-8$ & $9.9101 \mathrm{e}-8$ & $8.7645 \mathrm{e}-8$ & $6.9745 \mathrm{e}-8$ & $1.0292 \mathrm{e}-8$ & $4.0336 \mathrm{e}-9$ \\
\hline
\end{tabular}

Table4. Parameters extracted for the two-diode model

\begin{tabular}{l|llllll}
\hline & \multicolumn{3}{|c}{ Poly-crystalline } & \multicolumn{2}{c}{ Mono-crystalline } & \multicolumn{1}{c}{ Thin-Film } \\
\hline Module & KC200GT & S70 & SP70 & SQ150-PC & ST40 & PVL-136 \\
$I p v$ & 8.2100 & 4.5000 & 4.7000 & 4.8000 & 2.6800 & 5.1000 \\
$a_{1}$ & 1 & 1 & 1 & 1 & 1 & 1 \\
$a_{2}$ & 1.2000 & 1.2000 & 1.2000 & 1.2000 & 1.2000 & 1.2000 \\
$R s$ & 0.3300 & 0.3400 & 0.5100 & 0.9100 & 1.7100 & 1.9600 \\
$R s h$ & 174.1551 & 119.5882 & 94.9643 & 275.2625 & 204.8492 & 54.2497 \\
$I o_{l}=I o_{2}$ & $4.1280 \mathrm{e}-10$ & $4.9996 \mathrm{e}-10$ & $4.2065 \mathrm{e}-10$ & $3.1059 \mathrm{e}-10$ & $3.0748 \mathrm{e}-11$ & $7.5012 \mathrm{e}-12$ \\
\hline
\end{tabular}

Tables 5-8 show the relative errors for $\mathrm{P}_{\max }$, Voc and Isc at varying irradiance and temperature of SP70 and ST40 modules. The relative error is defined as:

$$
E_{\text {relative }}(X)=\left(\frac{a b s\left(X_{\text {data }}-X_{\text {calcul }}\right)}{X_{\text {data }}}\right) * 100
$$

The irradiance is maintained constant at STC. From the data it can be concluded, more accurate results are obtained from the two-diode model for the crystalline silicon technologies. 
Table5. Relative errors of three models at different irradiances $\left(\mathrm{T}=25^{\circ} \mathrm{C}\right)$ for SP70 module.

\begin{tabular}{|c|c|c|c|c|c|c|c|c|}
\hline $\begin{array}{l}\text { Irradiance } \\
\left(\mathrm{W} / \mathbf{m}^{2}\right)\end{array}$ & Parameters & Measured data & 4-P model & $\begin{array}{c}\text { Error } \\
\% \\
\end{array}$ & $\begin{array}{l}\text { 5-P } \\
\text { model } \\
\end{array}$ & $\begin{array}{l}\text { Error } \\
\% \\
\end{array}$ & $\begin{array}{l}\mathrm{D} \\
\text { model }\end{array}$ & $\begin{array}{c}\text { Error } \\
\% \\
\end{array}$ \\
\hline 1000 & $\begin{array}{l}\text { Pmax } \\
\text { Voc } \\
\text { Isc }\end{array}$ & $\begin{array}{l}70.07 \\
21.33 \\
4.682\end{array}$ & $\begin{array}{l}70.5 \\
21.39 \\
4.7\end{array}$ & $\begin{array}{l}0.61 \\
0.28 \\
0.38\end{array}$ & $\begin{array}{l}70.11 \\
21.35 \\
4.7\end{array}$ & $\begin{array}{l}0.057 \\
0.094 \\
0.38\end{array}$ & $\begin{array}{l}70.22 \\
21.34 \\
4.675\end{array}$ & $\begin{array}{l}0.21 \\
0.047 \\
0.15\end{array}$ \\
\hline 800 & $\begin{array}{l}\text { Pmax } \\
\text { Voc } \\
\text { Isc }\end{array}$ & $\begin{array}{l}56.13 \\
21.03 \\
3.752\end{array}$ & $\begin{array}{l}57.61 \\
21.18 \\
3.76\end{array}$ & $\begin{array}{l}2.64 \\
0.71 \\
0.21\end{array}$ & $\begin{array}{l}55.95 \\
21.07 \\
3.76\end{array}$ & $\begin{array}{l}0.32 \\
0.19 \\
0.21\end{array}$ & $\begin{array}{l}56.38 \\
21.13 \\
3.74\end{array}$ & $\begin{array}{l}0.45 \\
0.48 \\
0.32\end{array}$ \\
\hline 600 & $\begin{array}{l}\text { Pmax } \\
\text { Voc } \\
\text { Isc }\end{array}$ & $\begin{array}{l}41.89 \\
20.5 \\
2.815\end{array}$ & $\begin{array}{l}43.96 \\
20.91 \\
2.82\end{array}$ & $\begin{array}{l}4.94 \\
2.00 \\
0.18\end{array}$ & $\begin{array}{l}41.46 \\
20.72 \\
2.82\end{array}$ & $\begin{array}{l}1.026 \\
1.073 \\
0.18\end{array}$ & $\begin{array}{l}41.99 \\
20.84 \\
2.805\end{array}$ & $\begin{array}{l}0.24 \\
1.66 \\
0.36\end{array}$ \\
\hline 400 & $\begin{array}{l}\text { Pmax } \\
\text { Voc } \\
\text { Isc }\end{array}$ & $\begin{array}{l}27.53 \\
19.92 \\
1.882\end{array}$ & $\begin{array}{l}29.62 \\
20.53 \\
1.88\end{array}$ & $\begin{array}{l}7.59 \\
3.06 \\
0.11\end{array}$ & $\begin{array}{l}26.76 \\
20.19 \\
1.88\end{array}$ & $\begin{array}{l}2.79 \\
4.92 \\
0.11\end{array}$ & $\begin{array}{l}27.12 \\
20.43 \\
1.87\end{array}$ & $\begin{array}{l}1.49 \\
2.56 \\
0.64\end{array}$ \\
\hline 200 & $\begin{array}{l}\text { Pmax } \\
\text { Voc } \\
\text { Isc }\end{array}$ & $\begin{array}{l}13.17 \\
19.12 \\
0.9472\end{array}$ & $\begin{array}{l}14.72 \\
19.81 \\
0.94\end{array}$ & $\begin{array}{l}11.76 \\
3.61 \\
0.76\end{array}$ & $\begin{array}{l}12.08 \\
19.25 \\
0.94\end{array}$ & $\begin{array}{l}8.28 \\
0.68 \\
0.76\end{array}$ & $\begin{array}{l}11.99 \\
19.65 \\
0.935\end{array}$ & $\begin{array}{l}8.96 \\
2.77 \\
1.29\end{array}$ \\
\hline
\end{tabular}

Table6: Relative errors of three models at different temperatures $\left(E=1000 \mathrm{~W} / \mathrm{m}^{2}\right)$ for SP70 module.

\begin{tabular}{|c|c|c|c|c|c|c|c|c|}
\hline $\begin{array}{l}\text { Temperature } \\
\left({ }^{\circ} \mathbf{C}\right)\end{array}$ & Parameters & $\begin{array}{l}\text { Measured } \\
\text { data }\end{array}$ & $\begin{array}{l}\text { 4-P } \\
\text { model }\end{array}$ & $\begin{array}{l}\text { Error } \\
\%\end{array}$ & $\begin{array}{l}5-P \\
\text { model }\end{array}$ & $\begin{array}{l}\text { Error } \\
\%\end{array}$ & $\begin{array}{l}2 \mathrm{D} \\
\text { model }\end{array}$ & $\begin{array}{l}\text { Error } \\
\%\end{array}$ \\
\hline 20 & $\begin{array}{l}\text { Pmax } \\
\text { Voc } \\
\text { Isc }\end{array}$ & $\begin{array}{l}71.54 \\
21.71 \\
4.743\end{array}$ & $\begin{array}{l}72.23 \\
21.77 \\
4.69\end{array}$ & $\begin{array}{l}0.96 \\
0.28 \\
1.12\end{array}$ & $\begin{array}{l}71.76 \\
21.70 \\
4.69\end{array}$ & $\begin{array}{l}0.31 \\
0.046 \\
1.12\end{array}$ & $\begin{array}{l}71.82 \\
21.70 \\
4.665\end{array}$ & $\begin{array}{l}0.39 \\
0.046 \\
1.64\end{array}$ \\
\hline 40 & $\begin{array}{l}\text { Pmax } \\
\text { Voc } \\
\text { Isc }\end{array}$ & $\begin{array}{l}64.77 \\
20.18 \\
4.736\end{array}$ & $\begin{array}{l}65.29 \\
20.26 \\
4.73\end{array}$ & $\begin{array}{l}0.80 \\
0.39 \\
0.13\end{array}$ & $\begin{array}{l}65.15 \\
20.25 \\
4.73\end{array}$ & $\begin{array}{l}0.59 \\
0.35 \\
0.13\end{array}$ & $\begin{array}{l}65.38 \\
20.24 \\
4.705\end{array}$ & $\begin{array}{l}0.94 \\
0.29 \\
0.65\end{array}$ \\
\hline 60 & $\begin{array}{l}\text { Pmax } \\
\text { Voc } \\
\text { Isc }\end{array}$ & $\begin{array}{l}57.94 \\
18.71 \\
4.743\end{array}$ & $\begin{array}{l}58.34 \\
18.69 \\
4.77\end{array}$ & $\begin{array}{l}0.69 \\
0.11 \\
0.57\end{array}$ & $\begin{array}{l}58.54 \\
18.68 \\
4.77\end{array}$ & $\begin{array}{l}1.036 \\
0.16 \\
0.57\end{array}$ & $\begin{array}{l}58.86 \\
18.67 \\
4.745\end{array}$ & $\begin{array}{l}1.59 \\
0.21 \\
0.042\end{array}$ \\
\hline
\end{tabular}

Table7. Relative errors of three models at different irradiances $\left(\mathrm{T}=25^{\circ} \mathrm{C}\right)$ for ST40 module.

\begin{tabular}{|c|c|c|c|c|c|c|c|c|}
\hline $\begin{array}{l}\text { Irradiance } \\
\left(\mathrm{W} / \mathrm{m}^{2}\right)\end{array}$ & Parameters & Measured data & 4-P model & $\begin{array}{l}\text { Error } \\
\%\end{array}$ & $\begin{array}{l}5-P \\
\text { model }\end{array}$ & $\begin{array}{l}\text { Error } \\
\%\end{array}$ & $\begin{array}{l}\mathrm{DD} \\
\text { model }\end{array}$ & $\begin{array}{l}\text { Error } \\
\%\end{array}$ \\
\hline 1000 & $\begin{array}{l}\text { Pmax } \\
\text { Voc } \\
\text { Isc }\end{array}$ & $\begin{array}{l}40.21 \\
23.29 \\
2.677\end{array}$ & $\begin{array}{l}40.03 \\
23.30 \\
2.68\end{array}$ & $\begin{array}{l}0.45 \\
0.04 \\
0.11\end{array}$ & $\begin{array}{l}39.99 \\
23.27 \\
2.68\end{array}$ & $\begin{array}{l}0.55 \\
0.086 \\
0.11\end{array}$ & $\begin{array}{l}40.04 \\
23.26 \\
2.658\end{array}$ & $\begin{array}{l}0.42 \\
0.13 \\
0.71\end{array}$ \\
\hline 800 & $\begin{array}{l}\text { Pmax } \\
\text { Voc } \\
\text { Isc }\end{array}$ & $\begin{array}{l}31.71 \\
22.85 \\
2.149\end{array}$ & $\begin{array}{l}33.04 \\
23.02 \\
2.144\end{array}$ & $\begin{array}{l}4.19 \\
0.74 \\
0.23\end{array}$ & $\begin{array}{l}32.68 \\
22.99 \\
2.144\end{array}$ & $\begin{array}{l}3.06 \\
0.61 \\
0.23\end{array}$ & $\begin{array}{l}32.97 \\
23.04 \\
2.126\end{array}$ & $\begin{array}{l}3.97 \\
0.83 \\
1.07\end{array}$ \\
\hline 600 & $\begin{array}{l}\text { Pmax } \\
\text { Voc } \\
\text { Isc }\end{array}$ & $\begin{array}{l}23.52 \\
22.33 \\
1.607\end{array}$ & $\begin{array}{l}25.44 \\
22.67 \\
1.608\end{array}$ & $\begin{array}{l}8.16 \\
1.52 \\
0.062\end{array}$ & $\begin{array}{l}24.80 \\
22.62 \\
1.608\end{array}$ & $\begin{array}{l}5.44 \\
1.30 \\
0.062\end{array}$ & $\begin{array}{l}25.17 \\
22.76 \\
1.595\end{array}$ & $\begin{array}{l}7.02 \\
1.92 \\
0.75\end{array}$ \\
\hline 400 & $\begin{array}{l}\text { Pmax } \\
\text { Voc } \\
\text { Isc }\end{array}$ & $\begin{array}{l}15.34 \\
21.63 \\
1.074\end{array}$ & $\begin{array}{l}17.26 \\
22.17 \\
1.072\end{array}$ & $\begin{array}{l}12.52 \\
2.49 \\
0.19\end{array}$ & $\begin{array}{l}16.4 \\
22.11 \\
1.072\end{array}$ & $\begin{array}{l}6.91 \\
2.22 \\
0.19\end{array}$ & $\begin{array}{l}16.7 \\
22.35 \\
1.063\end{array}$ & $\begin{array}{l}8.86 \\
3.33 \\
1.02\end{array}$ \\
\hline
\end{tabular}




\begin{tabular}{l|llllllll}
\hline & & & & & & & & \\
\multirow{2}{200}{} & Pmax & 6.967 & 8.611 & 23.59 & 7.615 & 9.30 & 7.655 & 9.87 \\
& Voc & 20.28 & 21.33 & 5.18 & 21.17 & 4.39 & 21.61 & 6.56 \\
& Isc & 0.537 & 0.536 & 0.19 & 0.536 & 0.19 & 0.5316 & 1.01 \\
\hline
\end{tabular}

Table8: Relative errors of three models at different temperatures $\left(E=1000 \mathrm{~W} / \mathrm{m}^{2}\right)$ for ST40 module

\begin{tabular}{|c|c|c|c|c|c|c|c|c|}
\hline $\begin{array}{l}\text { Tempetaure } \\
\left({ }^{\circ} \mathrm{C}\right)\end{array}$ & Parameters & $\begin{array}{l}\text { Measured } \\
\text { data }\end{array}$ & $\begin{array}{l}4-P \\
\text { model }\end{array}$ & $\begin{array}{l}\text { Error } \\
\%\end{array}$ & $\begin{array}{l}5-P \\
\text { model } \\
\end{array}$ & $\begin{array}{l}\text { Error } \\
\% \\
\end{array}$ & $\begin{array}{l}\mathrm{DD} \\
\text { model }\end{array}$ & $\begin{array}{l}\text { Error } \\
\% \\
\end{array}$ \\
\hline 20 & $\begin{array}{l}\text { Pmax } \\
\text { Voc } \\
\text { Isc }\end{array}$ & $\begin{array}{l}41.29 \\
23.65 \\
2.702\end{array}$ & $\begin{array}{l}41.36 \\
23.80 \\
2.678\end{array}$ & $\begin{array}{l}0.33 \\
0.63 \\
0.89\end{array}$ & $\begin{array}{l}41.27 \\
23.76 \\
2.678\end{array}$ & $\begin{array}{l}0.048 \\
0.46 \\
0.89\end{array}$ & $\begin{array}{l}41.3 \\
23.75 \\
2.656\end{array}$ & $\begin{array}{l}0.024 \\
0.42 \\
1.70\end{array}$ \\
\hline 40 & $\begin{array}{l}\text { Pmax } \\
\text { Voc } \\
\text { Isc }\end{array}$ & $\begin{array}{l}36.36 \\
21.7 \\
2.702\end{array}$ & $\begin{array}{l}36.09 \\
21.79 \\
2.685\end{array}$ & $\begin{array}{l}0.74 \\
0.41 \\
0.63\end{array}$ & $\begin{array}{l}36.19 \\
21.77 \\
2.685\end{array}$ & $\begin{array}{l}0.47 \\
0.32 \\
0.63\end{array}$ & $\begin{array}{l}36.29 \\
21.75 \\
2.663\end{array}$ & $\begin{array}{l}0.19 \\
0.23 \\
1.44\end{array}$ \\
\hline 60 & $\begin{array}{l}\text { Pmax } \\
\text { Voc } \\
\text { Isc }\end{array}$ & $\begin{array}{l}31.49 \\
19.87 \\
2.706\end{array}$ & $\begin{array}{l}30.93 \\
19.77 \\
2.692\end{array}$ & $\begin{array}{l}1.78 \\
0.50 \\
0.52\end{array}$ & $\begin{array}{l}31.21 \\
19.76 \\
2.692\end{array}$ & $\begin{array}{l}0.89 \\
0.55 \\
0.52\end{array}$ & $\begin{array}{l}31.34 \\
19.75 \\
2.67\end{array}$ & $\begin{array}{l}0.48 \\
0.60 \\
1.33\end{array}$ \\
\hline
\end{tabular}

Figure. 6 and 8 shows the analysis for relative error of Voc and the $\mathrm{P}_{\max }$ for ST40 module at different irradiance levels. As can be seen at STC irradiance, there is a very small difference in the Voc values among the three models. However as the irradiance is reduced, there is a significant deviation of Voc calculated using the 4-P, 5-P and two-diode models. Similar results can be observed for the $\mathrm{P}_{\max }$.

Figure.7 shows the performance of the three models at different temperature for ST40 module. There is no significant difference between three models for Voc. However the four-parameter model exhibits poor performance for $\mathrm{P}_{\max }$ calculations.

Figure.9 shows the performance of the three models at different temperature for SP70 module.

We note that the two-diode model and the fiveparameter model are the least accurate at the three remarkable points at $60{ }^{\circ} \mathrm{C}$ compared to the fourparameter models. This is logical because the value of the ideality factor is assumed to be fixed in the fiveparameter model and the two-diode model and in the other hand the values of the recombination and diffusion saturation current are assumed to be equal in the two-diode model.

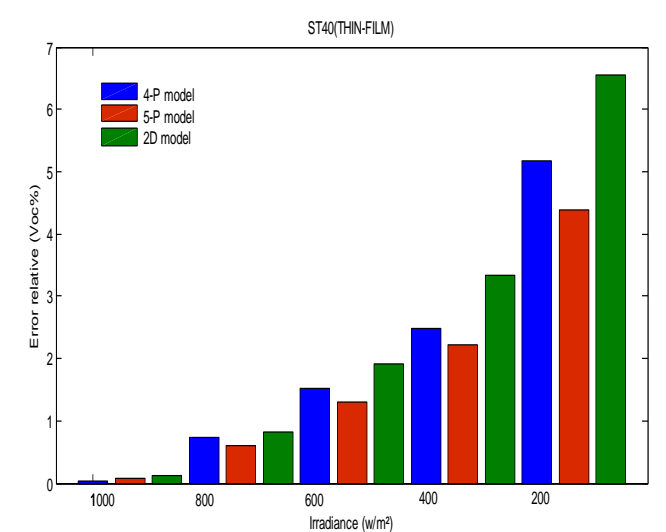

Figure.6. Relative error of $\mathrm{P}_{\max }$ (a) and Voc (b) at varying irradiance for ST40 PV module 

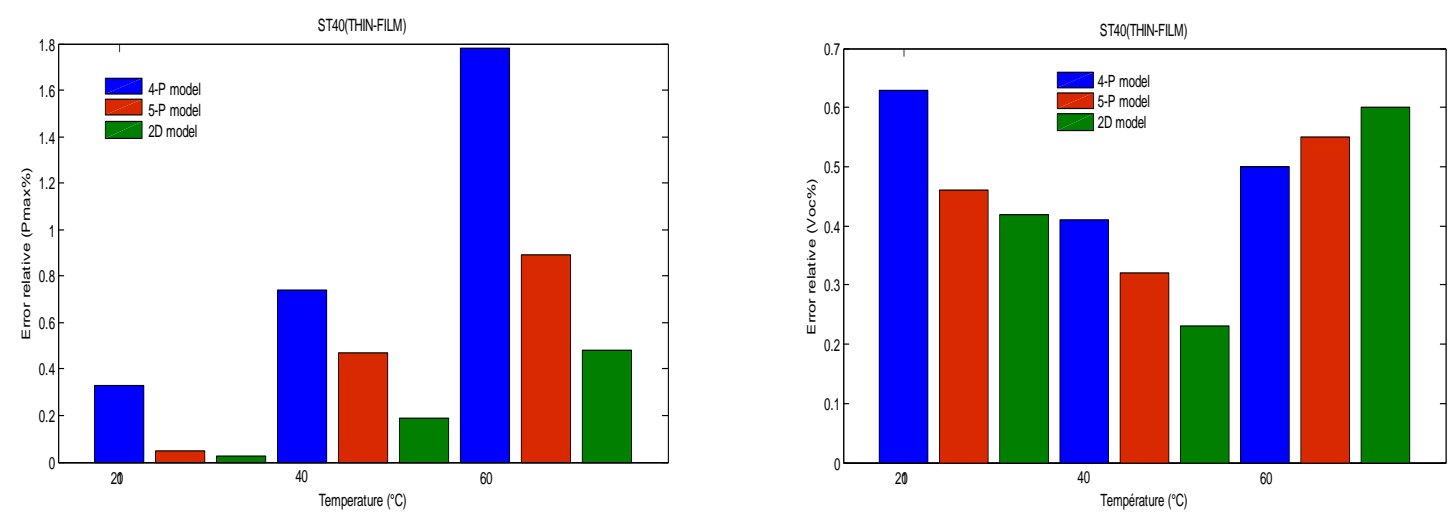

Figure.7. Relative error of $\mathrm{P}_{\max }$ (a) and Voc (b) at varying temperature for ST40 PV module
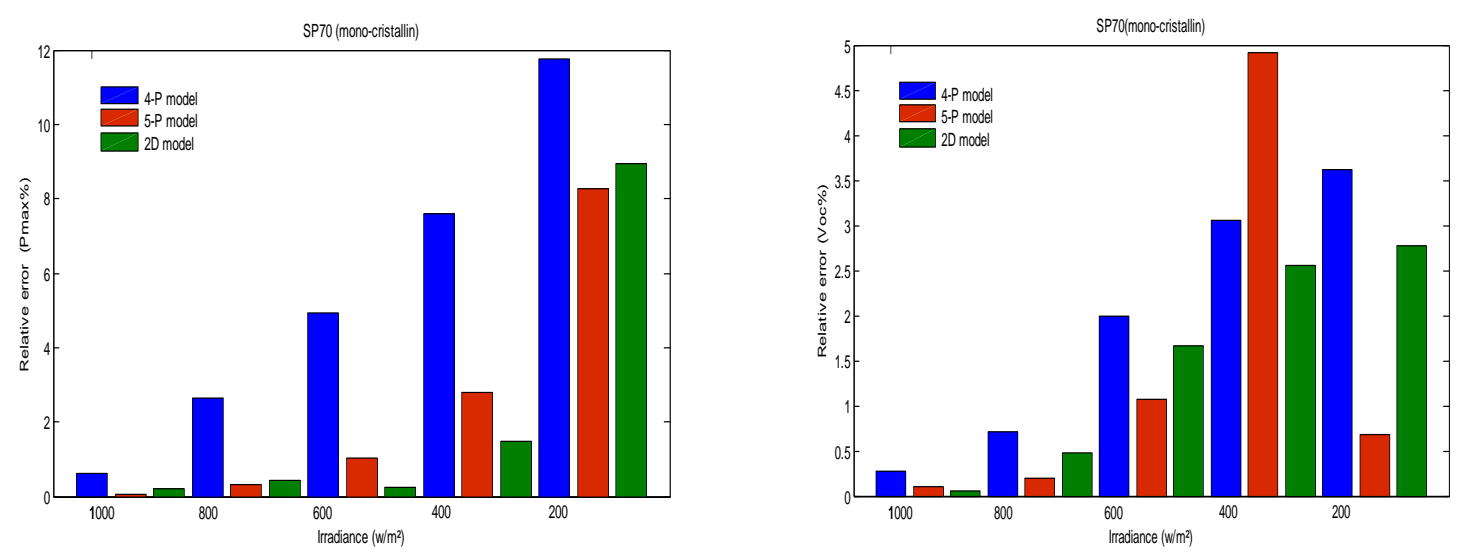

Figure.8. Relative error of $\mathrm{P}_{\max }$ (a) and Voc (b) at varying irradiance for SP70 PV module
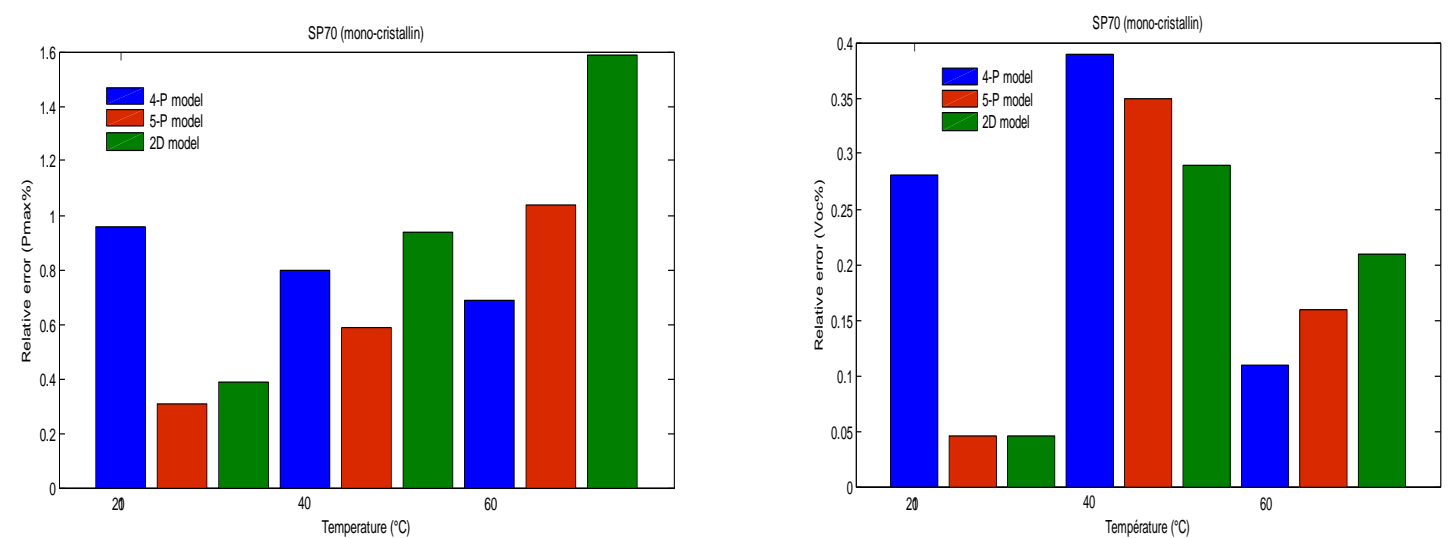

Figure.9. Relative error of $\mathrm{P}_{\max }$ (a) and Voc (b) at varying temperature for SP70 PV module

\section{Conclusion}

The present paper has proposed the comparison between the four-parameter, five-parameter and towdiode models. These models used to predict the electrical response of illuminated six PV modules for various operating conditions. The accuracy of the three models is evaluated using practical data from manufacturers of different types of PV modules. Its performances are compared with the experimental values given by the constructors. It has been found that, the tow-diode model is better when subjected to variations in irradiance and temperature.
And gives better accuracy for reconstructing the electrical characteristics of mono-crystalline and poly-crystalline PV modules, but for thin-film PV module the five parameter model is closer to the experimental data at the low irradiance.

\section{References}

[1] W. Durisch, D. Tille, A. Worz, and W. Plapp, "Characterisation ". of photovoltaic generators," Applied Energy, vol. 65, no. 1-4, 2000, pp. 273-284.

[2] A. Q. Jakhrani, A. K. Othman, A. R. H. Rigit, S. R. Samo, and S. R. Kamboh, "A novel analytical model 
for optimal sizing of standalone photovoltaic systems," Energy, vol. 46, no. 1,2012, pp. 675-682.

[3] A. N. Celik, "A simplifed model based on clearness index for estimating yearly performance of hybrid pv energy systems," Progress in Photovoltaics: Research and Applications, vol. 10, no. 8, 2002, pp. 545-554.

[4] A. H. Fanney and B. P. Dougherty, "Building integrated photovoltaic test facility," Journal of Solar Energy Engineering, vol. 123, no. 3, 2001, pp. 194199.

[5] M. A. Green, "Short communication: price/efciency correlations for 2004 photovoltaic modules," Progress in Photovoltaics: Research and Applications, vol. 13, 2005, pp. 85-87.

[6] Glass M. C., 'Improved Solar Array Power Point Model with SPICE Realization of Generalized', IECEC, Vol. 1,1996, pp. 286-291.

[7] Kajihara A and Harakawa A. T., 'Model of Photovoltaic Cell Circuits Under Partial Shading', ICIT, 2005, pp. 866-870.

[8] Tan Y. T., Kirschen D. S., and Jenkins N., 'A Model of PV Generation Suitable for Stability Analysis', IEEE Trans. Energy Converts, Vol. 19, No. 4, 2004, pp. 748755.

[9] A. Chatterjee, A. Keyhani, and D. Kapoor, "Identification of Photovoltaic Source Models," Energy Conversion, IEEE Transactions on, vol. PP, 2011, pp1-7.

[10] Celik, A. N. and Acikgoz, N. "Modelling and experimental verification of the operating current of mono-crystalline photovoltaic modules using four- and five-parameter models", Applied Energy, 84, 2007, pp $1-15$.

[11] Arab, A. H., Chenlo, F., Benghanem, M.,. "Loss- ofload probability of photovoltaic water pumping systems", Solar Energy, 76, 2004, pp713-723.

[12] Phang JCH, Chan DSH, Philips JR. Accurate analytical method for the extraction of solar-cell model parameters. Electron Letters, 20, 1984, pp406-408.
[13] Blas MA, Torres JL, Prieto E, Garcia A. Selecting a suitable model for characterizing photovoltaic devices. Renewable Energy, 25, 2002, pp371-380.

[14] Kou Q, Klein A, Beckman WA. A method for estimating the long-term performance of directcoupled PV pumping systems. Solar Energy, 64, 1998, pp 33-40.

[15] Chenlo F, Fabero F, Alonso MC. Testing, norms, reliability and harmonization: a comparative study between indoor and outdoor measurement. Agencia EUREC, Joule. JOUII-CT92-0178, Brussels: European Commission, 1996.

[16] Marcelo G. Villalva, Jonas R. Gazoli, and Ernesto R. Filho, "Comprehensive Approach to Modeling and Simulation of Photovoltaic Arrays ", IEEE Transactions on Power Electronics, 24, 2009, pp 1198 - 1208.

[17] Villalva, M.G., Gazoli, J.R., Filho, E.R. Comprehensive approach to modeling and simulation of photovoltaic arrays. IEEE Trans. Power Electron. 24, 2009, pp1198-1208

[18] Ishaque, K., Salam, Z., Taheri, H., Shamsudin, A. A critical evaluation of EA computational methods for photovoltaic cell parameter extraction based on two diode model. Sol. Energy, 85, 2011, pp 1768-1779.

[19] Walker, G.R.: Evaluating MPPT topologies using a Matlab PV model. Journal of Electrical \& Electronics Engineering, 21, 2001, pp 49-56.

[20] Ishaque, K., Salam, Z., Taheri, H. Simple, fast and accurate two diode model for photovoltaic modules. Sol. Energy Mater. Sol. Cells, 95 (2), 2011a, pp 586594.

[21] Villalva, M.G., Gazoli, J.R., Filho, E.R. Comprehensive approach to modeling and simulation of photovoltaic arrays. IEEE Trans. Power Electron. 24, 2009, pp 1198-1208. 